\title{
Status update of the MACE Gamma-ray telescope
}

\author{
K. K. Yadav ${ }^{a, b, *}$ on behalf of the HiGRO Collaboration \\ (a complete list of authors can be found at the end of the proceedings) \\ ${ }^{a}$ Astrophysical Sciences Division, Bhabha Atomic Research Centre, \\ Mumbai 400094, India \\ ${ }^{b}$ Homi Bhabha National Institute, \\ Mumbai 400094, India \\ E-mail: kkyadav@barc.gov.in
}

MACE (Major Atmospheric Cherenkov Experiment), an imaging atmospheric Cherenkov telescope, has recently been installed by the HiGRO (Himalayan Gamma-Ray Observatory) collaboration at Hanle $\left(32.8^{\circ} \mathrm{N}, 78.9^{\circ} \mathrm{E}, 4270 \mathrm{~m}\right.$ asl) in Ladakh region of North India. The telescope has a $21 \mathrm{~m}$ diameter large light collector consisting of indigenously developed 1424 square-shaped diamond turned spherical aluminum mirror facets of size $\sim 0.5 \mathrm{~m} \times 0.5 \mathrm{~m}$. MACE is the second largest Cherenkov telescope at the highest altitude in the northern hemisphere. The imaging camera of the telescope consists of 1088 photo-multiplier tubes with a uniform pixel resolution of $\sim 0.125^{\circ}$ covering a field of view of $\sim 4.0^{\circ} \times 4.0^{\circ}$. The main objective of the MACE telescope is to study gamma-ray sources mainly in the unexplored energy region $20-100 \mathrm{GeV}$ and beyond with high sensitivity. In this paper, we describe the key design features and current status of MACE including results from the trial observations of the telescope.

$37^{\text {th }}$ International Cosmic Ray Conference (ICRC 2021)

July 12th - 23rd, 2021

Online - Berlin, Germany

\footnotetext{
*Presenter
} 


\section{Introduction}

MACE (Major Atmospheric Cherenkov Experiment), a very high energy (VHE) $\gamma$-ray telescope based on imaging atmospheric Cherenkov technique, is currently under commissioning at Hanle, India [1, 2]. The Indian astronomical site at Hanle $\left(32.8^{\circ} \mathrm{N}, 78.9^{\circ} \mathrm{E}, 4270 \mathrm{~m}\right.$ asl $)$ in the Himalayan range is at the highest altitude in the world for any existing imaging atmospheric Cherenkov telescope (IACT) at present. The main objective of the MACE telescope is the observation of the VHE $\gamma$-ray photons with energy $30 \mathrm{GeV}$ and above, which is traditionally considered as the window for spacebased telescopes. Observations with the MACE telescope will help in understanding the production and propagation of energetic $\gamma$-ray photons in the non-thermal Universe and to also explore some phenomena which are not accessible to terrestrial accelerators. Some of the important astrophysical motivations for the MACE telescope include: discovery of new $\gamma$-ray sources, identification of unassociated sources in the high energy Fermi catalog, detection of the $\gamma$-ray emission from various types of known sources (active galactic nuclei, gamma-ray bursts, pulsars and so on) at different ages of the Universe, providing evidence for the enigmatic jet formation process etc. Beyond probing the non-thermal Universe and cosmic accelerators, the VHE observations using the MACE telescope are also expected to address a range of cosmological topics such as measurement of the intensity of extragalactic background light, cosmic ray electron spectrum, search for nature of dark matter candidates like weakly interacting massive particles [3-5].

\section{The MACE Telescope: Salient Features}

The MACE telescope is equipped with a $21 \mathrm{~m}$ diameter quasi-parabolic reflector with $\mathrm{f} / \mathrm{d}$ ratio of $\sim 1.2$. The quasi-parabolic design of such a large reflector helps in reducing the optical aberrations of the telescope. To achieve such a large light collector area, reflector of the MACE telescope is segmented into 1424 small, square-shaped spherical mirror facets of size $0.488 \mathrm{~m} \times$ $0.488 \mathrm{~m}$ each with varying focal lengths (Figure $1($ Left)). Four such mirrors with similar focal length are mounted on a single panel of size $0.986 \mathrm{~m} \times 0.986 \mathrm{~m}$ each and a total of 356 mirror panels with varying focal length in the range $25 \mathrm{~m}$ to $26.2 \mathrm{~m}$ are obtained. All the 356 mirror panels mounted on the telescope basket are manually aligned in such a way that the resulting surface behaves like a single quasi-parabolic reflector with focal length gradually increasing from the center of the basket towards the periphery. An active mirror alignment control system is used for orienting each mirror panel to achieve the desired optical quality of the reflector. The metallic mirror facets are made up of Aluminium alloy supported by a honeycomb structure. Diamond turning technology [6] has been used to fabricate all the mirror facets in order to achieve desired mirror quality in terms of surface finish and accuracy within the country (Figure 1(Left)). The reflectance of these mirror facets is greater than $85 \%$ in the wavelength range $280-700 \mathrm{~nm}$. Due to its large size, the MACE telescope is not protected by a dome and the mirrors are continuously exposed to the environment. The reflecting surface of each mirror facet is coated with a thin $(100-150 \mathrm{~nm})$ layer of $\mathrm{SiO}_{2}$ for protecting the reflecting surface and ensuring its longevity.

The telescope deploys a 1088-pixel imaging camera with resolution of $0.125^{\circ}$ at its focal plane, covering a field of view of $4.36^{\circ} \times 4.03^{\circ}$. Six stage photomultiplier tubes (pixel) with 38 

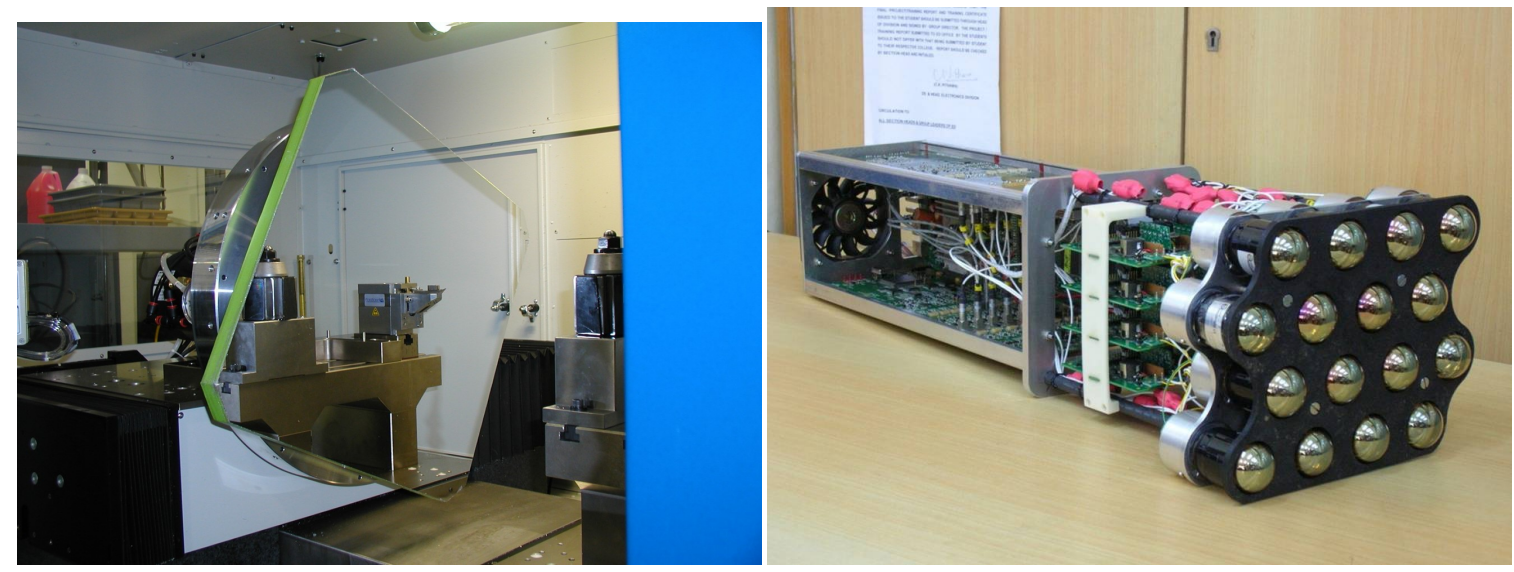

Figure 1: Left: A diamond turned mirror facet for the MACE light collector. Right: 16-channel camera integrated module for the camera of MACE telescope.

mm diameter arranged at a triangular pitch of $55 \mathrm{~mm}$ are used in the MACE-camera for detection of the Cherenkov photons. Each photomultiplier tube is provided with a hexagoanl front-coated light concentrator in order to enhance the light collection efficiency by collecting the Cherenkov photons incident in the dead space between adjacent pixels. The light collection efficiency of these compound parabolic concentrators is more than $85 \%$ in the wavelength range of 230-600 nm. The MACE-camera is modular in design and consists of 68 camera integrated modules (CIMs) of 16 channels each. Each module channel has its signal processing electronics (photomultiplier tubes with programmable high voltage generators, pre-amplifiers, amplifiers, discriminators, first level trigger generation logic, and signal digitization circuitry) housed in it (Figure 1(Right)). An analogue switched capacitor array DRS-4 is used as ring sampler at $1 \mathrm{GHz}$ speed for continuous digitization of the signal from the photomultiplier tubes. The signal from the photomultiplier tubes is simultaneously amplified at low and high gain of 14 and 140 respectively to ensure a large dynamic range. The discriminator output amplitude of each channel is used for monitoring its single channel rate and also for generating the first-level trigger from an individual CIM. The first-level triggers from all the modules are collated in a second-level trigger generator where proximity of the triggered pixels in adjacent modules is checked. The innermost 576 pixels $(24 \times 24)$ are used for generating the trigger according to predefined logic for nearest pairs, triplets, quadruplets, etc of the pixels. After the generation of the second-level trigger, the data from all the 68 modules are collected by the data concentrator, which in turn sends them to the data acquisition computer in the control room through optical fibres. It is expected that about 50GB of data will be stored during every hour of observation with the MACE telescope [7, 8].

An altitude-azimuth mount drive system of the MACE telescope provides the stability of $21 \mathrm{~m}$ diameter large mechanical structure with 180 ton weight by using a track and wheel design. Two azimuth drive wheels are coupled to three phase, permanent magnet brushless AC servo motors through multi-stage gearboxes for providing the azimuth motion. The elevation movement is provided through a gearbox coupled to a 13-section bull-gear assembly of $11.6 \mathrm{~m}$ radius. All the drives are provided with counter torque capability to avoid gear backlash error. The motors are driven by pulse width modulated drive amplifiers powered by 480 Volt DC from a solar power 


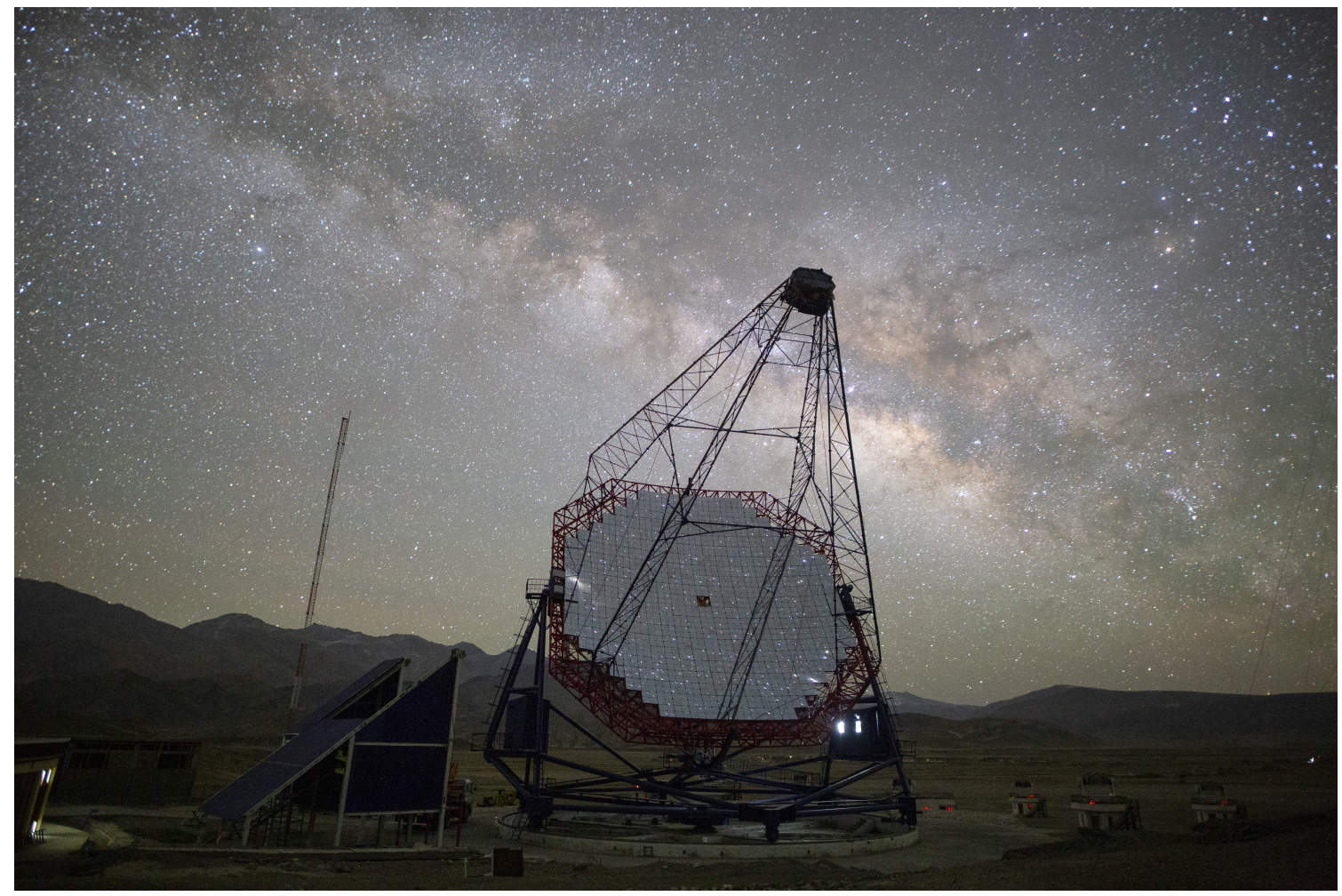

Figure 2: The MACE telescope installed at Hanle-Ladakh, India.

station. The positions of the two axes are monitored by a 25-bit absolute optical encoders with $\sim 20$ arcsec accuracy. Both the azimuth and elevation gear boxes also have high-speed options to move the telescope at speed of $3^{\circ} s^{-1}$ to quickly point the telescope in the direction of transient events like gamma-ray bursts in the sky. The MACE drive system can provide tracking accuracy of better than 1 arcmin in wind speeds of up to $30 \mathrm{~km} \mathrm{~h}^{-1}$. The telescope is automatically brought to the parking position if the sustained wind speed is more than $40 \mathrm{~km} \mathrm{~h}^{-1}$. Moreover, the telescope structure is designed for survival at a wind speed of $150 \mathrm{~km} \mathrm{~h}^{-1}$.

\section{Present Status of the MACE Telescope}

The installation of the MACE telescope at Hanle site was successfully completed in October 2020. A recent picture of the telescope at the site is depicted in Figure 2. The trial runs for testing the performance of different components of the fully assembled telescope started in November 2020. Monte Carlo simulation studies carried out using the CORSIKA package suggest that the MACE telescope is expected to have a $\gamma$-ray trigger energy threshold of $\sim 20 \mathrm{GeV}$ in the low zenith angle range below $40^{\circ}$ [9]. At high zenith angles above $40^{\circ}$, the threshold energy increases to $\sim 150$ $\mathrm{GeV}$. The dynamic energy range of the MACE telescope is expected to be $20 \mathrm{GeV}-5 \mathrm{TeV}$ with a total trigger rate of $\sim 1 \mathrm{kHz}$. The integral sensitivity for a point source with Crab Nebula like spectrum 


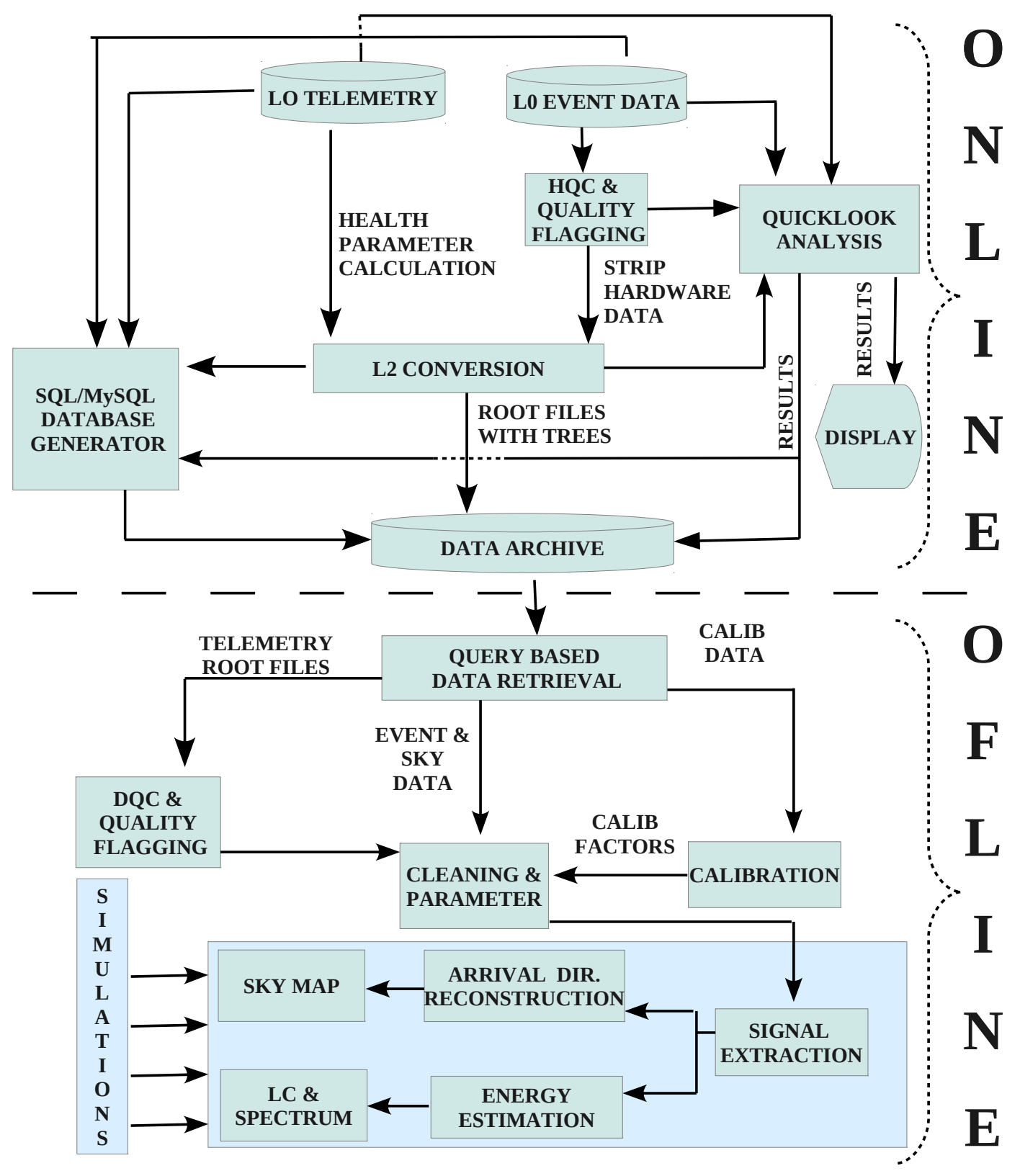

Figure 3: Flowchart for the MACE data Analysis Package (MAP). 

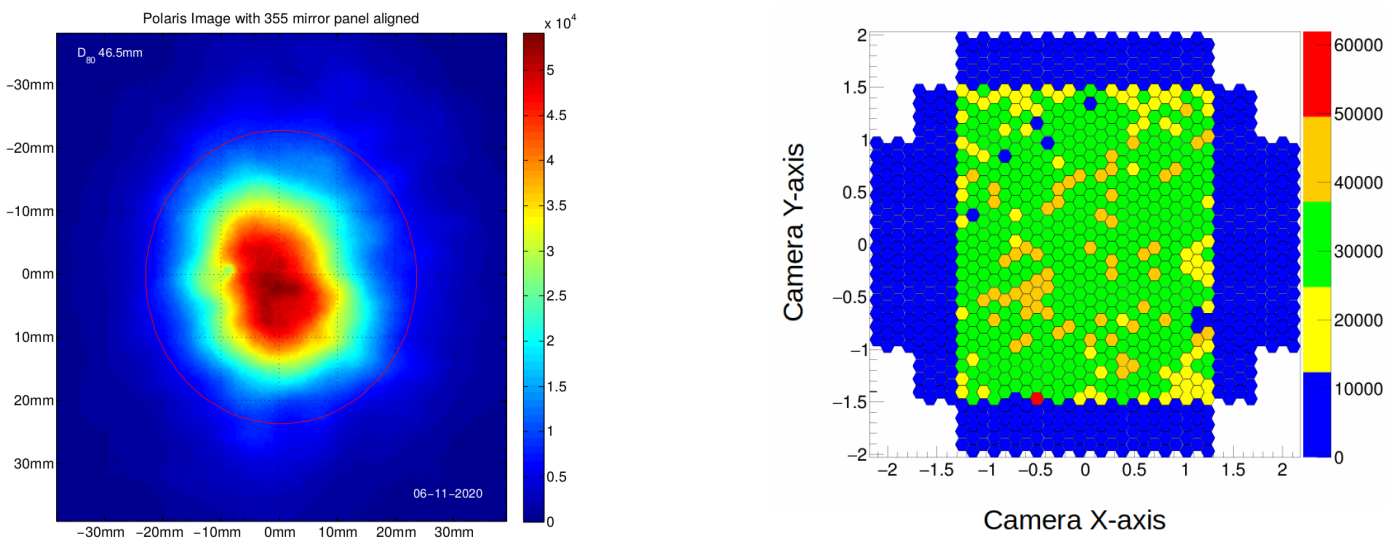

Figure 4: Left: The point spread function of the MACE telescope measured for Polaris. Right: Hit pattern uniformity in the trigger region of the camera during the Crab Nebula observations for $\sim 1$ hour on April 1 , 2021.

above $30 \mathrm{GeV}$ is $2.7 \%$ at $5 \sigma$ statistical significance level in 50 hours of observation [10]. The angular resolution of MACE telescope is estimated as $\sim 0.21^{\circ}$ in the energy range of $30-50 \mathrm{GeV}$ and this improves to $\sim 0.06^{\circ}$ in the energy band $1.8-3 \mathrm{TeV}[11]$. The telescope is expected to have energy resolutions of $\sim 40 \%$ and $\sim 19 \%$ in the energy bands $30-50 \mathrm{GeV}$ and $1.8-3 \mathrm{TeV}$ respectively. For an effective analysis of the real time data collected with the MACE telescope, a data analysis software called MAP (MACE Analysis Package) has been developed. The MAP represents collection of ROOT-based programs/routines written in C-language under LINUX platform. Different analysis steps included in the MAP are outlined in Figure 3. Another version of the MAP based on Python libraries is also under development.

\section{Preliminary Results}

The MACE telescope, currently under its commissioning phase, has been deployed for special trail runs since October 2020. Alignment of the 356 mirror panels on telescope basket is carried out with the help of an active mirror alignment control system while tracking the pole star. A CCD camera along with $180 \mathrm{~mm}$ focal length lens mounted on the center of the telescope basket is used to capture the reflected image of an optical source on telescope focal plane. The image of pole star captured by the CCD camera on the focal plane after background subtraction and image cleaning is shown in Figure 4(Left). D80 (defined as the diameter of circle, concentric with the centroid of the image, which contains $80 \%$ intensity of the image) of the image is found to be $46.5 \mathrm{~mm}$ after performing a Gaussian fit to the pole star image data. This characterizes the point sperad function of the telescope.

In order to record the Cherenov event data, the information of hit channels in the camera of the telescope is also stored. The number of hits for each triggered pixel in the trigger region (inner 576 pixels) of the camera during the trial run in the direction of the Crab Nebula on the night of April 1, 


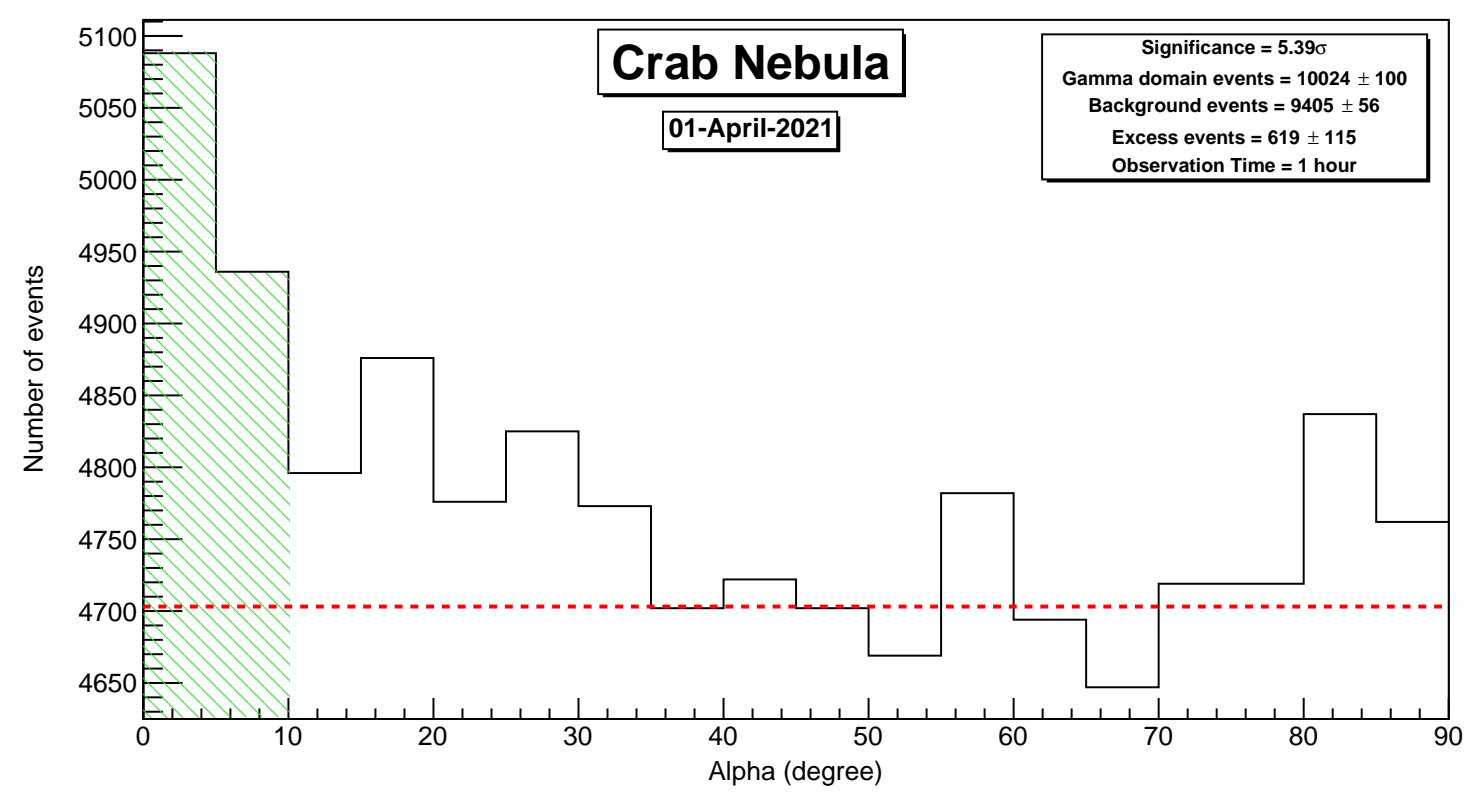

Figure 5: Preliminary results from the Crab Nebula observations on April 1, 2021 for a live time of $\sim 1$ hour.

2021 for about 1 hour of observation is reported in Figure 4(Right). This indicates the homogeneity of the camera of the MACE telescope in generating the trigger in response to the Cherenkov events.

Preliminary analysis of the data collected on the Crab Nebula on April 1, 2021 has been performed using the standard Hillas Parameterization method [12] implemented in the MAP. The distribution of the Hillas orientation parameter $(\alpha)$ for the recorded Cherenkov events is depicted in Figure 5. The $\alpha$-plot shown here only represents the initial performance of the MACE telescope without standard data quality checks for IACT observations. Although there is an indication of the presence of $\gamma$-ray like events in the source region at $\sim 5.4 \sigma$ statistical significance level in Figure 5, yet these preliminary results in the present form cannot be referred to as the first light of the MACE telescope. A detailed analysis of the data collected on the Crab Nebula with MACE in the month of April 2021 is currently underway and the final results of the first light are expected very soon.

\section{Summary}

A group of very high energy $\gamma$-ray astronomers are actively involved in setting up the MACE telescope in India under HiGRO (Himalayan Gamma Ray Observatory) Collaboration. The construction and installation of the telescope at Hanle-site has been successfully completed. Currently, the MACE telescope is under its commissioning phase and its first light is expected very soon. Subsequently, the telescope will enter into the science operatione phase. Once fully operational, the MACE telescope, equipped with a $21 \mathrm{~m}$ diameter light collector and 1088-pixel camera, will be the second largest $\gamma$-ray telescope (after the Large Size Telescope prototype (LST-1) at the Cherenkov Telescope Array) in the northern hemisphere. However, altitude of the MACE-site is the highest in the world. This geographical advantage along with the large light collector will help in exploring 
the $\gamma$-ray sky above $15 \mathrm{GeV}$ and also provide an excellent energy overlap with the space-based $\gamma$-ray observatories.

\section{Acknowledgements}

The HiGRO collaboration thanks all the colleagues from various Divisions at Bhabha Atomic Research Centre, who have been involved at different stages of the construction and installation of the MACE telescope at Hanle, India.

\section{References}

[1] R. Koul, MACE gamma-ray telescope-a status update, CURRENT SCIENCE 113 (2017) 691

[2] K. K. Singh, \& K. K. Yadav, 20 Years of Indian Gamma Ray Astronomy Using Imaging Cherenkov Telescopes and Road Ahead, Universe 7 (2021) 96

[3] F. Aharonian et al., High energy astrophysics with ground-based gamma ray detectors, Reports on Progress in Physics 71 (2008) 096901

[4] A. M. Hillas, Evolution of ground-based gamma-ray astronomy from the early days to the Cherenkov Telescope Arrays, Astroparticle Physics 43 (2013) 19

[5] G. Di Sciascio, Ground-based Gamma-Ray Astronomy: an Introduction, Journal of Physics Conference Series 1263 (2019) 012003

[6] T. Sugano et al., Diamond Turning of an Aluminum Alloy for Mirror, CIRP Annals 36 (1987) 17

[7] S. Srivastava et al., MACE camera controller embedded software: Redesign for robustness and maintainability, Astronomy and Computing 30 (2020) 100358

[8] D. Sarkar et al., Comparison of data storage and analysis throughput in the light of high energy physics experiment MACE, Astronomy and Computing 33 (2020) 100409

[9] C. Borwankar et al., Simulation studies of MACE-I: Trigger rates and energy thresholds, Astroparticle Physics 84 (2016) 97

[10] M. Sharma et al., Sensitivity estimate of the MACE gamma ray telescope, Nuclear Instruments and Methods in Physics Research A 851 (2017) 125

[11] C. Borwankar et al., Estimation of expected performance for the MACE $\gamma$-ray telescope in low zenith angle range, Nuclear Instruments and Methods in Physics Research A 953 (2020) 163182

[12] A. M. Hillas, Cerenkov Light Images of EAS Produced by Primary Gamma Rays and by Nuclei, 19th International Cosmic Ray Conference 3 (1985) 445 


\section{Full Authors List: HiGRO Collaboration}

N. Bhatt ${ }^{1}$, S. Bhattacharyya ${ }^{1,2}$, C. Borwankar ${ }^{1}$, K. Chanchalani ${ }^{1}$, P. Chandra ${ }^{1}$, V. R. Chitnis ${ }^{3}$, N. Chouhan ${ }^{1}$, M. P. Das ${ }^{1}$, V. K. Dhar ${ }^{1}$, B. Ghosal ${ }^{1}$, S. Godambe ${ }^{1}$, S. Godiyal ${ }^{1}$, K. K. Gour ${ }^{1}$, J. Hariharan $^{1}$, M. Khurana ${ }^{1}$, M. Kothari ${ }^{1}$, S. Kotwal ${ }^{1}$, M. K. Koul ${ }^{1}$, N. Kumar ${ }^{1}$, N. Kumar ${ }^{1}$, C. P. Kushwaha $^{1}$, N. Mankuzhiyil ${ }^{1}$, P. Marandi ${ }^{1}$, K. Nand ${ }^{1}$, S. Norlha ${ }^{1}$, D. Sarkar ${ }^{1}$, M. Sharma ${ }^{1}$, K. K.

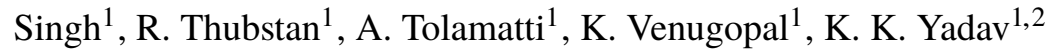

${ }^{1}$ Astrophysical Sciences Division, Bhabha Atomic Research Centre, Mumbai 400094, India.

${ }^{2}$ Homi Bhabha National Institute, Anushakti Nagar,Mumbai 400094, India.

${ }^{3}$ Department of High Energy Physics, Tata Institute of Fundamental Research, Homi Bhabha Road, Colaba, Mumbai 400005, India. 